\title{
Primary Care Patients' and Providers' Perspectives about an Online Weight Management Program: a Qualitative Study
}

\author{
Ronen Rozenblum, PhD, MPH ${ }^{1,2}$, Barbara A. De La Cruz, BA ${ }^{7}$, Nyryan V. Nolido, MA' , \\ Ihorma Adighibe, $R N^{7}$, Kristina Secinaro, $R D, M S^{7}$, Katherine D. McManus, RD, $M S^{7}$, \\ Florencia Halperin, MD, MMSc ${ }^{7,3}$, Jason P. Block, MD, MPH $H^{1,3,4}$, \\ David W. Bates, MD, MSc $c^{1,3,5}$, and Heather J. Baer, $S C D^{1,3,5}$
}

\begin{abstract}
'Brigham and Women's Hospital, Boston, MA, USA; ${ }^{2}$ Division of General Internal Medicine and Primary Care \& Harvard Medical School, Boston, MA, USA; ${ }^{3}$ Harvard Medical School, Boston, MA, USA; ${ }^{4}$ Harvard Pilgrim Health Care Institute, Boston, MA, USA; ${ }^{5}$ Harvard School of Public Health, Boston, MA, USA.
\end{abstract}

BACKGROUND: Primary care providers (PCPs) often take the lead role in caring for patients with overweight and obesity; however, few PCPs counsel patients about weight loss. Online weight management programs that are integrated within primary care may help address this gap in care.

OBJECTIVE: To identify perceptions of and experience with online weight management programs in general and with a proposed online program, to identify barriers to use, and to improve the design and content of our intervention, which included an online program plus population health management (PHM) support from primary care practices. DESIGN: A mixed qualitative methods study including three patient focus groups and seven semi-structured interviews with healthcare providers.

PARTICIPANTS: A total of 13 adult patients (age range, 20-70) with body mass index (BMI) $27-35 \mathrm{~kg} / \mathrm{m}^{2}$ attended the focus groups. In-person semi-structured interviews were conducted with seven healthcare providers (three PCPs, two population health managers, one primary care nurse, and one registered dietitian).

MAIN MEASURES: We developed and used semistructured focus groups and interview guides. The focus group and interviews were recorded and transcribed. Using grounded theory, we analyzed the transcripts to identify and extract common concepts and themes.

KEY RESULTS: Although patients and healthcare providers expressed positive opinions about online weight management programs, few patients had experience with them, and providers stated that such programs are not being widely implemented in primary care settings. Some participants highlighted the flexibility and low cost as strengths of online weight management tools compared with in-person programs. All participants had favorable opinions about our proposed intervention and were overwhelmingly positive about the combination of an online program and PHM support.

CONCLUSIONS: This study highlights the potential value of online weight management programs and PHM support in primary care.

CLINICAL TRIALS REGISTRATION: NCT02656693.

Received February 19, 2019

Revised April 8, 2019

Accepted April 9, 2019

Published online May 31, 2019
KEY WORDS: weight management; overweight; obesity; primary care; online program.

J Gen Intern Med 34(8):1503-21

DOI: $10.1007 / \mathrm{s} 11606-019-05022-6$

(c) Society of General Internal Medicine 2019

\section{INTRODUCTION}

Over two thirds of US adults have overweight or obesity, defined as body mass index $(\mathrm{BMI}) \geq 25 \mathrm{~kg} / \mathrm{m}^{2} .{ }^{1}$ Overweight and obesity are associated with many serious health conditions, including type 2 diabetes, cardiovascular disease, and some cancers. ${ }^{2-5}$ Even small amounts of weight loss can lead to significant health benefits, and a variety of weight loss strategies have been shown to be effective. ${ }^{6-10}$ Primary care providers (PCPs) can play an important role in helping patients with weight management. ${ }^{11-13}$ Clinical practice guidelines recommend that PCPs offer or refer their patients to an intensive, multi-component behavioral intervention if they would benefit from weight loss. ${ }^{10}$ However, PCPs often do not have sufficient time or training to counsel patients about weight, and other services may be difficult for patients to access due to convenience and cost. ${ }^{1-20}$

Advances in patient-facing health information technology (HIT) have changed the landscape of self-management. ${ }^{21} \mathrm{Al}-$ though data increasingly support the role of these tools in improving health services' efficiency and health outcomes, ${ }^{22-24}$ several studies to date have shown mixed results on the effect of these technologies on quality of care. ${ }^{25}$ Currently, health centers perceive patient-facing HIT as an ideal tool to engage their patient populations in chronic disease management. ${ }^{26}$

Evidence from a number of studies, including several within the primary care setting, indicates that online (i.e., Internetbased) weight management programs can help people achieve and maintain clinically meaningful weight loss, ${ }^{27-39}$ However, these programs are not being widely implemented in primary care, and it is unclear whether they are effective or scalable in real clinical practice. ${ }^{39}$ To address this, we conducted the Partnerships for Reducing Overweight and Obesity with Patient-Centered Strategies (PROPS) Study. The overall goal 
of the PROPS Study was to adapt an evidence-based online weight management program, integrate it with population health management (PHM) support from primary care practices, and conduct a cluster-randomized trial to compare the effectiveness of the combined intervention (including the online program and PHM support) with the online weight management program alone and with usual care. The study design and methods for the trial are described elsewhere (Baer et al., 2019; in preparation.)

This paper describes the methods and results of the qualitative work conducted during the first phase of the study, namely focus groups with patients and semi-structured interviews with healthcare providers, to obtain feedback about the proposed intervention before the main trial began. We aimed to identify perceptions of and experience with online weight management programs in general and with our proposed online program, identify barriers to use, and iteratively improve the design and content of the intervention.

\section{METHODS}

BMIQ is a cloud-based, HIPAA-compliant platform designed for healthcare providers to deliver weight management programs to patients in a variety of settings. ${ }^{40-42}$ For the PROPS Study, we made several enhancements to the existing BMIQ application; these are described in detail elsewhere (Baer et al., 2019; in preparation). Currently, BMIQ offers patients 33 educational sessions, delivered over a period of 12 months; these sessions are available in written and video formats and cover a variety of topics, including healthy eating, physical activity, and goal setting. To complement these sessions, the program also includes structured exchange-based meal plans; tools for tracking food, physical activity, and weight; and tailored feedback via email and text messages (see Fig. 1 for key BMIQ features). ${ }^{40} \mathrm{~A}$ professional interface in BMIQ allows providers to monitor patients' engagement with the program, including their logins, sessions viewed, and tracking data. ${ }^{40}$ In our study, population health managers (PHMs), who are non-clinical staff working closely with the primary care practices, and research staff used the professional interface to monitor patients' progress, conduct outreach, and provide periodic updates to PCPs. BMIQ is available in both English and Spanish and can be accessed via a computer, tablet, or smartphone.

To obtain patient and provider feedback about weight management programs and tools before starting the trial, we conducted three patient focus groups and seven semi-structured interviews with healthcare providers during March and April of 2016, several months prior to the start of recruitment for the main trial. The study took place at Brigham and Women's Hospital (BWH) in Boston, MA, and was reviewed and approved by the Partners HealthCare Human Research Institutional Review Board (protocol \# 2015P002372). The PROPS trial was also registered on clinicaltrials.gov (ID: NCT02656693). All of the patients and healthcare providers who participated in the focus groups and interviews provided verbal informed consent.

\section{Focus Groups with Patients}

Study Design, Aims, and Focus Group Guides. Two focus group guides were developed based on current literature and expert opinion: one guide for the first two focus groups (Appendix 1, online) and a second guide for the third group (Appendix 2, online). We piloted both of these guides with several patient and stakeholder advisors, who have provided advice throughout the course of the study, and refined them based on their feedback. The aim of the first two focus groups was to solicit general feedback from patients about weight management programs and about our proposed intervention. The aim of the third focus group, which was comprised of a subset of patients who had already participated in one of the first two focus groups, was to obtain more specific feedback about BMIQ after patients had used it on their own.

Participants. Eligibility criteria for the focus groups were similar to those for the PROPS main trial. Patients were between 20 and 70 years old, had BMI between 27 and $35 \mathrm{~kg} / \mathrm{m}^{2}$, were English-speaking, had access to the Internet, and were interested in weight management and/or motivated to lose weight. We identified patients using data from the electronic health record and recommendations from PCPs.

We identified 112 eligible patients for the focus groups. We contacted 47 of these patients following the PCPs' approval; we screened 34, and 23 of these patients were determined to be eligible. In total, 13 participated in the focus groups; five participated in one of the two initial focus groups as well as the third, which was designed to collect more specific feedback after patients had used the BMIQ program on their own. Table 1 summarizes the characteristics of the focus group participants.

\section{Semi-structured Interviews with Providers}

Study Design, Aims, and Interview Guide. We conducted semi-structured interviews with healthcare providers to gather their perspectives about weight management programs and tools available to patients and their opinions about our proposed intervention. To do so, we created a semi-structured interview guide (Appendix 3, online) that was developed based on a literature review and expert opinion.

Participants. We identified seven BWH healthcare providers who are considered key stakeholders. We conducted in-person 


\section{BMIQ Key Features}
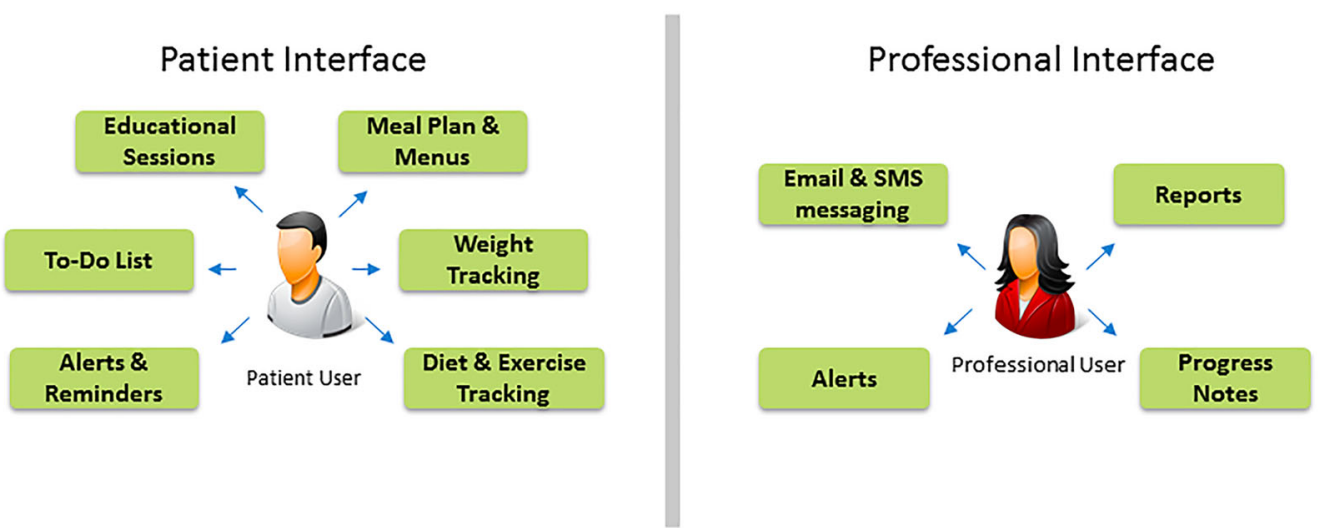

Figure 1 Illustration of the BMIQ Program.

interviews with three PCPs, two PHMs, one primary care registered nurse, and one registered dietitian.

Data Collection and Analysis. The focus groups and interviews were recorded and transcribed with the consent of the participants. Using grounded theory, ${ }^{43}$ we analyzed the transcripts to identify and extract common concepts and themes.

\section{RESULTS}

\section{Focus Groups with Patients}

Perceptions of and Previous Experience with Weight Management Programs. While most focus group participants had tried to lose weight in the past, only a few had used formal weight management programs, including online weight management tools. The participants who had previous experience with formal weight loss programs had tried Weight Watchers and/or used online tools like Loselt!, MyFitnessPal, and SparkPeople. Most participants who had used these programs reported positive opinions about them and felt that they had helped them to lose weight. Specifically, they mentioned that programs enhanced their awareness and motivation to lose weight and taught them how to change their eating habits and lifestyle. For example, one person stated that:

I don't even really like to call it [Weight Watchers] a diet as much as it's just a change in your lifestyle... and it really taught me to be more careful about portion control.

Participants highlighted the barriers and limitations of these programs. They emphasized that it can be hard to stick with the programs (e.g., to keep tracking points/calories and change their daily routine and eating habits). They also noted that weight loss is a long and difficult process. Finally, one informant stated that he had stopped using a program because the recommended meals were too expensive. Below are representative comments from the participants:

I think for me,... what's not as successful is I don't keep up with the exercise, and,...I snack too much. I'm snacking and I know that would be too many points if I were actually keeping track of the points, but I don't keep track of the points anymore, and so... it's, it's hard, because you have to continually work at the program. It's hard to follow plans with Weight Watchers, anyway, I mean, it's like... you know, there's so much food out there that... it's bad for ya? But it's still so good that you want to eat it anyway, regardless!

I participated in Weight Watchers. Initially it was great. I lost the weight I wanted to lose and maintained that for quite some time...and then I kind of fell off the wagon and, then I lost weight again, on Weight Watchers, and then I fell off the wagon again, and... that's where I am now.

Participants also noted that most tools are cumbersome and often require a lot of work to track items, limiting regular use. For example, one person stated that:

I got one...it does a lot of tracking of your activity. So, that's good. And it gives you a calorie count - just how much you're burning. But you can also go online and enter your food in there, too, but I haven't... it's just an added thing. 
Table 1 Characteristics of the Focus Group Participants

\begin{tabular}{|c|c|c|}
\hline Characteristics & $\begin{array}{l}\text { Number of participants } \\
(N=13)\end{array}$ & $\begin{array}{l}\text { Percentage } \\
(\%)\end{array}$ \\
\hline \multicolumn{3}{|l|}{ Gender } \\
\hline Male & 3 & 23.1 \\
\hline Female & 10 & 76.9 \\
\hline \multicolumn{3}{|l|}{ Age } \\
\hline $21-30$ & 1 & 7.7 \\
\hline $31-40$ & 0 & 0 \\
\hline $41-50$ & 3 & 23.1 \\
\hline $51-60$ & 4 & 30.8 \\
\hline $61-70$ & 5 & 38.5 \\
\hline \multicolumn{3}{|l|}{ Race } \\
\hline Black or African- & 6 & 46.1 \\
\hline \multicolumn{3}{|l|}{ American } \\
\hline White & 4 & 30.8 \\
\hline Other & 1 & 7.7 \\
\hline Not reported & 2 & 15.4 \\
\hline \multicolumn{3}{|l|}{ Education } \\
\hline 8th grade or less & 1 & 7.7 \\
\hline High school graduate or & 2 & 15.4 \\
\hline \multicolumn{3}{|l|}{ GED } \\
\hline $\begin{array}{l}\text { Some college or 2-year } \\
\text { degree }\end{array}$ & 6 & 46.1 \\
\hline 4-year college graduate & 1 & 7.7 \\
\hline $\begin{array}{l}\text { More than 4-year de- } \\
\text { gree }\end{array}$ & 3 & 23.1 \\
\hline \multicolumn{3}{|l|}{ Self-reported health status } \\
\hline Poor or fair & 4 & 30.8 \\
\hline Good & 6 & 46.1 \\
\hline Very good or excellent & 3 & 23.1 \\
\hline
\end{tabular}

\section{Attitudes Towards Proposed Intervention}

General Feedback About the Program. We received general feedback about our proposed intervention in the first two focus groups and more specific feedback from the five participants who participated in the third focus group after using and testing the online program on their own. Overall, the participants (in all focus groups) seemed to like BMIQ; they felt it looked simple and easy to follow and found all of the features important for weight management.

The majority of participants felt that the tracking tools were the most important to use. Participants stated that using BMIQ made them think more about their diet and health. They thought they would use all of the features at first, but as soon as they figured out which ones were the most useful for them, they might use just a few of them. The opinions of participants on how often they would use BMIQ ranged from two to five times per week.

While most participants who tested BMIQ on their own used a desktop computer to access the website, some also used laptops, tablets, and smartphones. Several participants accessed BMIQ in more than one way. Surprisingly, very few participants used smartphones to access BMIQ. This could be because most of the participants were in their $50 \mathrm{~s}$ and $60 \mathrm{~s}$; in addition, the program was not de- signed as a mobile application. See below for some representative comments from the participants.

The phone is too small for me to do it.

Well, I thought maybe that I'd try to use my mobile phone ... because that's quicker for me, and then I have it with me all the time,... whenever I'm sitting' down, whatever I'm doing', I could just... even when I'm out, I can just go through,.....and check it out. But... it didn't work for me, though.

Finally, the majority of participants felt that the integration of BMIQ with PHM support could significantly help study participants manage their weight. Specifically, they liked the idea that a PHM would be reaching out to patients at certain times to provide feedback and support. Most participants also stated that they feel comfortable talking about weight management with someone other than their primary care doctor (e.g., a PHM or dietitian).

Feedback About Specific Features Within the Program. The following findings (Table 2) are related to specific features within BMIQ. This feedback is based on specific tasks that the participants were asked to do when they tested the program on their own (Appendix 2, online).

\section{Semi-structured Interviews with Providers} Feedback About Weight Management Programs in Primary Care. When asked about available weight management programs, the majority mentioned several BWH weight management programs, such as the "surgical management program" and the "medical weight management program," but none in their primary care practices. They stated that if needed, they refer their patients to other programs at BWH and/or to their registered dietitian.

The registered dietitian we interviewed stated that her department's activities in the ambulatory care setting are not as comprehensive as in the inpatient setting. Their main ambulatory activity is a referral service for patients; the department also teaches primary care physicians about nutrition and weight management. She stated that one of her main goals is to better engage primary care physicians in weight management programs and initiatives.

With the exception of the registered dietitian, none of the healthcare providers who participated were actively engaged in weight management programs for patients, including research initiatives. The providers had mixed opinions about the extent to which these weight management programs were useful. Most agreed that the success of these programs really depends on the 
Table 2 Attitudes Towards Specific Features of BMIQ Program

\begin{tabular}{|c|c|}
\hline $\begin{array}{l}\text { Specific features of the BMIQ } \\
\text { program }\end{array}$ & Attitudes/key findings \\
\hline Home page & $\begin{array}{l}\text { - All participants felt that there was great value in the information displayed on the home page. } \\
\text { - Although most participants liked the home page, several people felt that it was overwhelming. }\end{array}$ \\
\hline Navigation from home page & $\begin{array}{l}\text { - All participants stated that navigation between tabs was easy, and they found what they expected } \\
\text { on each page. }\end{array}$ \\
\hline Sessions page & $\begin{array}{l}\text { - All participants thought that the educational materials on the sessions page (written information and } \\
\text { videos) were very clear and useful. } \\
\text { - The participants stated that the video length (5-10 min each) was appropriate. } \\
\text { - All participants believed that having both video and text was a good way to engage patients. }\end{array}$ \\
\hline Meal plan page & $\begin{array}{l}\text { - Participants liked the Meal Plan page; they thought it was practical and would be relatively easy to follow. } \\
\text { - They believed that there was a great value in the exchange-based meal plan, which gives them additional options } \\
\text { for items within the meal plan, and that it would lead to a higher rate of adherence. }\end{array}$ \\
\hline Weight page & $\begin{array}{l}\text { - All participants thought that the weight page and the visual presentation of their progress were very useful. } \\
\text { - Most participants thought that it is doable to enter weight } 2 \text { times per week. } \\
\text { good to give a range of healthy BMIs as well as the definition of BMI and the formula for calculating it. }\end{array}$ \\
\hline Food and activity page & $\begin{array}{l}\text { - Most participants thought that the Food and Activity tracking features could be useful. } \\
\text { - Participants had a mixed experience using tracking features on the phone. }\end{array}$ \\
\hline
\end{tabular}

patients themselves, with the most likely benefit among those who are extremely motivated and have resources.

According to the providers, one of the barriers to engaging patients in the current hospital programs is the fact that weight management programs are typically not covered by insurance. Thus, for many patients, these programs are very expensive. All of the providers stated that they were aware of other weight management programs that their patients were using outside their practices, especially Weight Watchers. Overall, they had a positive attitude towards these outside programs. They believed that if these programs promote physical activity, healthy eating, and gradual weight loss, then they are useful initiatives. A few providers worried that some of these programs were a "quick fix" and not sustainable. Most of the providers do not actively refer their patients to other weight management programs outside their practices but support the patients if they use such programs. For example, one PCP said, "I encourage my patients to do whatever works for them."

In general, most providers had positive attitudes towards online weight management tools and programs because of routine access to smartphones, which enable people with limited resources to have access to these programs. Specifically, they mentioned LoseIt! and MyFitnessPal as programs their patients had used successfully. Below are some representative comments from the providers:

My attitudes (towards the online weight management tools/programs) are pretty pragmatic. If it works for you, then it's great.

I think generally it's a great idea. You have your coach in your pocket.

\section{Specific Feedback About Our Proposed Intervention}

In general, providers felt that there was great value in having a reliable online program that providers can recommend to their patients. Specifically, they appreciated the various features of the online program. All providers were overwhelmingly positive about our proposed intervention. They believed that the integration of an online program with PHM support could be a powerful and effective weight management strategy. There was a consensus about the importance of the PHM role in this type of intervention- "The human touch," as one of the providers said. According to them, patients need this support to overcome problems and to feel that someone cares about them. For example, one provider stated that

You might feel accountable to your smartphone; but you might feel more accountable to your smartphone plus the population health managers that call you on

Tuesday. I think it's great!

All providers also saw great value in receiving information about their patients from this program. Some providers wanted to receive information about all their patients who engaged in the program and some only about ones who had issues (e.g., lost a lot of weight in a short time period). Most providers (especially the physicians) stated that they would like to receive a concise report about their patients. They wanted the report to include a list of their patients, how much weight they had lost, and if there were any important issues that they should know about (red flags). All providers thought that eventually they would like the information incorporated and viewable 
in the electronic health record (EHR); other options for receiving information were via email and/or meeting with the PHMs. Some thought that once per month is a reasonable time period for updates, and others preferred a time period of once every two to three months.

When asked if they were interested in having direct access to the patient online weight management program, the providers reported that they would not have the time to use it. The providers had mixed opinions about communicating with their patients via the online weight management program. Some stated that they encouraged patients to communicate with them via the patient portal within the EHR. Nevertheless, communicating with patients via a separate website (outside of the electronic health record) did not seem realistic to them.

\section{DISCUSSION}

We assessed perceptions among both patients and providers of experience with online weight management programs in general and with our proposed intervention (BMIQ online program integrated with PHM support). Our goal was also to identify strategies to overcome barriers to use and to iteratively improve the design and content of our intervention.

One key theme was that while most focus group participants had tried to lose weight previously, few had actually used formal weight management tools, even though most had positive impressions of them if they had previously used one. Interestingly, a few participants highlighted some strengths of the online weight management tools compared with in-person programs, stating that it is hard to follow through with the inperson programs, which could also be very expensive. Consistent with this notion, previous studies have shown that online weight management programs can help people achieve and maintain clinically meaningful weight loss, ${ }^{27-36}$ and that online programs may increase convenience and decrease the cost of lifestyle interventions. ${ }^{30,38}$

Our findings from the semi-structured interviews with healthcare providers confirm and extend the findings from the patient focus groups, as well as from previous studies that have examined the current state of weight management in primary care. ${ }^{44}$ The majority of providers stated that while hospitals have weight management programs, primary care settings do not widely implement such programs. Moreover, providers had mixed opinions regarding the extent to which in-person weight management programs were useful.

In addition, most of the providers in this study had positive attitudes towards online weight management tools and programs because of their accessibility. Consistently, several studies indicate that even though PCPs perceived having important roles in patients' successful weight management, they are not currently engaged in this process. ${ }^{14-20,45}$ Other studies have shown that online weight management programs can help patients achieve weight loss in the primary care setting ${ }^{30,39,46}$ and be cost-effective. ${ }^{47}$

Finally, both patients and providers seemed to like our proposed intervention. They saw great value in having a reliable online program such as BMIQ and found its features important for weight management. All participants in this study (i.e., patients and providers) were overwhelmingly positive about the combination of the online program with PHM support. They believed that the integration of BMIQ and PHM support could be a very powerful and effective weight management strategy. Thus, there was consensus about the importance of the PHM in this type of intervention.

Our study was limited to patients and providers who were associated with BWH or one of its affiliated primary care clinics, which limits the generalizability of our results. In addition, the patients and providers who chose to participate may not be representative of all patients and providers at BWH. Future studies examining perspectives in a broader group of patients and healthcare providers, both at BWH and at other institutions, would be helpful.

Our patient focus groups and semi-structured interviews with healthcare providers highlight the potential value of online weight management programs and PHM support in primary care settings. While patients and healthcare providers expressed positive opinions about online weight management programs, few patients had tried them, representing an opportunity for a welldesigned intervention. Providers confirmed that such programs are not being widely implemented in primary care settings. Thus, primary care clinics should consider developing and incorporating this type of program into their offerings and engage providers and patients in these important initiatives.

Acknowledgments: Research reported in this presentation was funded through a Patient-Centered Outcomes Research Institute (PCORI) Award (14-092-1758). All statements in this report, including its findings and conclusions, are solely those of the authors and do not necessarily represent the views of the Patient-Centered Outcomes Research Institute (PCORI), its Board of Governors, or its Methodology Committee.

Corresponding Author: Ronen Rozenblum, PhD, MPH; Division of General Internal Medicine and Primary Care \& Harvard Medical School, Boston, MA, USA (e-mail: rrozenblum@bwh.harvard.edu).

\section{Compliance with Ethical Standards:}

This study is compliant with Ethical Standards.

Conflict of Interest: The authors declare that they do not have a conflict of interest. 


\section{REFERENCES}

1. Flegal KM, Carroll MD, Kit BK, et al. Prevalence of obesity and trends in the distribution of body mass index among US adults, 1999-2010. JAMA 2012;307:491-7.

2. Overweight, obesity, and health risk. National Task Force on the Prevention and Treatment of Obesity. Arch Intern Med 2000;160:898904.

3. Flegal KM, Graubard BI, Williamson DF, et al. Excess deaths associated with underweight, overweight, and obesity. JAMA 2005;293:1861-7.

4. Ogden CL, Carroll MD, McDowell MA, et al. Obesity among adults in the United States no change since 2003-2004. Hyattsville: National Center for Health Statistics; 2007.

5. Ogden CL, Yanovski SZ, Carroll MD, et al. The epidemiology of obesity. Gastroenterology 2007; 132:2087-102.

6. National Institutes of Health. Clinical guidelines on the identification, evaluation, and treatment of overweight and obesity in adults-the evidence report Obes Res 1998;6 Suppl 2:51S-209S.

7. The prevention or delay of type 2 diabetes. Diabetes Care 2002;25:742-9.

8. Anderson JW, Konz EC. Obesity and disease management: effects of weight loss on comorbid conditions. Obes Res 2001;9 Suppl 4:326S-34S.

9. Franz MJ, VanWormer JJ, Crain AL, et al. Weight-loss outcomes: a systematic review and meta-analysis of weight-loss clinical trials with a minimum 1-year follow-up. J Am Diet Assoc 2007;107: 1755-67.

10. Jensen MD, Ryan DH, Apovian CM, et al. 2013 AHA/ACC/TOS guideline for the management of overweight and obesity in adults: a report of the American College of Cardiology/American Heart Association Task Force on Practice Guidelines and The Obesity Society. Circulation 2013.

11. Hunt JR, Kristal AR, White E, et al. Physician recommendations for dietary change: their prevalence and impact in a population-based sample. Am J Public Health 1995;85(5):722-6.

12. Campbell MK, DeVellis BM, Strecher VJ, et al. Improving dietary behavior: the effectiveness of tailored messages in primary care settings. Am J Public Health 1994;84(5):783-7.

13. Nawaz H, Adams ML, Katz DL. Weight loss counseling by health care providers. Am J Public Health 1999;89(5):764-7.

14. Foster GD, Wadden TA, Makris AP, et al. Primary care physicians attitudes about obesity and its treatment. Obes Res 2003;11:1168-77.

15. Lyznicki JM, Young DC, Riggs JA, et al. Obesity: assessment and management in primary care. Am Fam Physician 2001;63:2185-96.

16. Shay LE, Shobert JL, Seibert $\mathbf{D}$, et al. Adult weight management: translating research and guidelines into practice. J Am Acad Nurse Pract 2009;21:197-206.

17. Frank E, Kunovich-Frieze T. Physicians' prevention counseling behaviors: current status and future directions. Prev Med 1995;24:543-5.

18. Huang J, Yu H, Marin E, et al. Physicians' weight loss counseling in two public hospital primary care clinics. Acad Med 2004;79:156-61.

19. Logue EE, Smucker WD. Obesity management in primary care: changing the status quo. J Fam Pract 2001;50:520.

20. Wadden TA, Didie E. What's in a name? Patients' preferred terms for describing obesity. Obes Res 2003;11:1140-6.

21. Pew Research Center. 2014. Cell phone and smartphone ownership demographics URL: http://www.pewinternet.org/data-trend/mobile/ cell-phone-and-smartphone-ownership-demographics [accessed 201903-20]

22. Greene J, Hibbard JH, Sacks $\mathbf{R}$, et al. When patient activation levels change, health outcomes and costs change, too. Health Aff (Millwood) 2015;34(3):431-437.

23. Grando MA, Rozenblum R, Bates DW, editors. Information technology for patient empowerment in healthcare. 1st ed. Berlin: Walter de Gruyter Inc; 2015

24. Hibbard JH, Greene $\mathbf{J}$, Overton V. Patients with lower activation associated with higher costs; delivery systems should know their patients' 'scores'. Health Aff (Millwood) 2013 Feb;32(2):216-222.

25. Rozenblum R, Donzé J, Hockey PM, et al. The impact of medical informatics on patient satisfaction: a USA-based literature review. Int J Med Inform 2013;82(3):141-58.
26. Broderick A, Haque F. Mobile health and patient engagement in the safety net: a survey of community health centers and clinics. Issue Brief (Commonw Fund) 2015;9:1-9.

27. Coons MJ, Demott A, Buscemi J, et al. Technology interventions to curb obesity: a systematic review of the current literature. Curr Cardiovasc Risk Rep 2012;6: 120-34.

28. Harvey-Berino J, Pintauro S, Buzzell P, et al. Does using the Internet facilitate the maintenance of weight loss? Int J Obes Relat Metab Disord 2002;26: 1254-60.

29. Harvey-Berino J, Pintauro S, Buzzell P, et al. Effect of internet support on the long-term maintenance of weight loss. Obes Res 2004;12:320-9.

30. McTigue KM, Conroy MB. Use of the internet in the treatment of obesity and prevention of type 2 diabetes in primary care. Proc Nutr Soc 2013;72:98-108.

31. Neve M, Morgan PJ, Jones PR, et al. Effectiveness of web-based interventions in achieving weight loss and weight loss maintenance in overweight and obese adults: a systematic review with meta-analysis. Obes Rev 2009.

32. Norman GJ, Zabinski MF, Adams MA et al. A review of eHealth interventions for physical activity and dietary behavior change. Am J Prev Med 2007;33:336-45.

33. Saperstein SL, Atkinson NL, Gold RS. The impact of Internet use for weight loss. Obes Rev 2007;8:459-65.

34. Svetkey LP, Stevens VJ, Brantley PJ, et al. Comparison of strategies for sustaining weight loss: the weight loss maintenance randomized controlled trial. JAMA 2008;299:1139-48.

35. Weinstein PK. A review of weight loss programs delivered via the Internet. J Cardiovasc Nurs 2006;21:251-8;259-60.

36. Wing RR, Tate DF, Gorin AA, et al. A self-regulation program for maintenance of weight loss. N Engl J Med 2006;355:1563-71.

37. Bennett GG, Herring SJ, Puleo E, et al. Web-based weight loss in primary care: a randomized controlled trial. Obesity (Silver Spring) 2009.

38. Ahern DK, Phalen JM, Mockenhaupt RE. Science and the advancement of eHealth: a call to action. Am J Prev Med 2003;24:108-9.

39. Levine DM, Savarimuthu S, Squires A, et al. Technology-assisted weight loss interventions in primary care: a systematic review. J Gen Intern Med 2014.

40. BMIQ professional program. https://www.bmiq.com/. Accessed 20 March 2019.

41. Wolf A, Knox L, Meeks K, et al. Efficacy to effectiveness: translating the ICAN lifestyle intervention to the real world. Paper presented at: The 33rd Annual Meeting of The Obesity Society, Obesity Week2015; Los Angeles, California

42. Kornhauser $\mathbf{N}$, Welch $\mathbf{R}$, Cigler $\mathbf{T}$, et al. Feasibility and effectiveness of BMIQ: a novel web-based weight loss program in overweight/obese breast cancer survivors. Paper presented at: The 33rd Annual Meeting of The Obesity Sociey, Obesity Week2015; Los Angeles, California.

43. Strauss A, Corbin J. Basics of qualitative research: techniques and procedures for developing grounded theory. 2nd ed. Thousand Oaks: Sage; 1998.

44. Tsai AG, Remmert JE, Butryn ML, Wadden TA. Treatment of obesity in primary care. Med Clin North Am 2018;102(1):35-47.

45. Bennett WL, Gudzune KA, Appel LJ, et al. Insights from the POWER practice-based weight loss trial: a focus group study on the PCP's role in weight management. J Gen Intern Med 2014;29(1):50-8.

46. Appel LJ, Clark JM, Yeh HC, et al. Comparative effectiveness of weightloss interventions in clinical practice. N Engl J Med. 2011;365(21):19591968

47. Little P, Stuart B, Hobbs FR, et al. An internet-based intervention with brief nurse support to manage obesity in primary care (POWeR+): a pragmatic, parallel-group, randomised controlled trial. Lancet Diabetes Endocrinol 2016:4(10):821-828.

Publisher's Note Springer Nature remains neutral with regard to jurisdictional claims in published maps and institutional affiliations. 
APPENDIX 1. FOCUS GROUP GUIDE (FOCUS GROUPS 1 AND 2) FOCUS GROUPS: FOCUS GROUP GUIDE

Once again we would like to thank you for participating in this PCORI PROPS focus group. Before we begin, let's go around the room and introduce ourselves. (5min)

\section{General QUeStions (10min)}

The first set of questions will explore your own experience with weight management and weight management tools or programs.

1. Have you ever participated in a weight management program, such as Weight Watchers or Jenny Craig? If yes, what was your experience?

a. PROBE: In your own opinion, what makes a weight management program successful?

b. PROBE: In your own opinion, what makes a weight management program unsuccessful?

2. Have you ever used online weight management programs or tools, such as websites or apps for your phone or tablet? (May need verbal clarification)

3. What is your overall opinion of online weight management tools or programs?

a. PROBE: Do you think that they can successfully help users to lose weight?

b. PROBE: Are there certain resources or services that you think would be useful for you?

\section{BMIQ QUESTIONS (45min)}

This next set of questions will focus specifically on our proposed online weight management program, which is called BMIQ. Before we begin, I will give you a brief introduction to BMIQ. We will focus on reviewing a few of the main features of BMIQ, which include educational sessions, meal plans, and tools for tracking your food, physical activity, and weight.

*Introduce educational sessions* (15 $\mathrm{min})$

The educational sessions are one of the main components of BMIQ. The program will include approximately 32 sessions. Each session covers a different topic and includes written content as well as a video (about five minutes long) covering the same information. Sessions will be released to the participants on a weekly basis or every other week over the course of one year. *Show a video $(20 \mathrm{sec}) *$

Each session is accompanied by a To-Do list with items for the participant to complete on a weekly basis. 
*Show To-Do list*

1. What is your overall opinion of the educational sessions available on BMIQ?

a. PROBE: What do you like the most about the sessions?

b. PROBE: What do you like the least about the sessions?

2. In general, do you think they contain an appropriate amount of information?

a. PROBE: Do you think anything should be added?

b. PROBE: Do you think anything should be taken out?

3. Do you like the accompanying videos?

4. How often would you want sessions released to you? (e.g. Once a week, biweekly.)

5. What topics do you think are the most important to cover in the sessions?

6. What do you think of the To-Do lists?

a. PROBE: Do you think asking participants to complete several items on a weekly basis could be overwhelming?

7. Do you have any other suggestions for how we can improve the educational sessions or To-Do lists?

*Show screenshot of mobile version of BMIQ educational session*

This is a screenshot of what the BMIQ educational sessions will look like if accessed on a smartphone or tablet.

8. What is your opinion of the mobile version of the sessions?

a. PROBE: Is there anything you would like to see added to the mobile version?

b. PROBE: Is there anything you think is unnecessary or should be taken out of the mobile version?

*Introduce meal plan* (15min)

The next feature of BMIQ is the meal plan. The meal plan recommends eating "whole foods" such as vegetables, fruits, lean meats, low-fat dairy, beans, and whole grains. Calorie targets are given based on your starting weight. For each calorie goal, there are 14 days of sample menus. Each menu has suggestions for breakfast, lunch, dinner, and snacks. In addition, there is an exchange-based meal plan for each calorie goal. The exchange-based meal plan provides some options if you don't want to follow the sample menus exactly.

9. What is your overall opinion of the BMIQ meal plan?

a. PROBE: What do you like the most about the meal plan?

b. PROBE: What do you like the least about the meal plan? 
10. Do you think that you would be able to follow the meal plan?

a. PROBE: How easy or difficult do you think it would be to follow this meal plan?

11. What is your opinion of the exchange-based meal plan?

a. PROBE: Do you think you would be more likely to use the sample menus or the exchange-based meal plan?

b. PROBE: When/why do you think you would use sample menus or exchangebased menu plan?

12. Do you have any suggestions for how we can improve the meal plan?

a. PROBE: Based on your experience, how much do you think you would use the meal plan?

*Show screenshot of mobile version of the BMIQ meal plan and sample menus*

This is a screenshot of what the BMIQ meal plan and sample menus will look like if accessed on a smartphone or tablet.

13. What is your opinion of the mobile version of the meal plan and menus?

*Introduce tracking features - BMIQ website * (15min)

The last main feature of our online program is the tracking tools. Participants will be able to track their food intake, physical activity, and weight and will be encouraged to do this regularly. *Introduce online tools for tracking diet and activity *

For tracking their food and physical activity, participants will use the food and activity tracking tools in BMIQ. They will have direct access to these tools through the BMIQ website.

Information recorded by these food and physical activity tracking tools will be available to the population health managers, who work with the primary care practices, and members of the study staff so that they can monitor and conduct outreach when necessary.

*Introduce basic tracking tool*

Because we anticipate that some participants may not do detailed food and activity tracking on a regular basis, we will also offer a more basic "check-in" feature for participants to indicate how well they are doing in terms of following the program. If participants report any challenges, such as food shopping, there will be some suggestions provided to them.

*Introduce weight tracker*

A weight tracker is also available on BMIQ. Recorded weights will be shown in the graph so that participants can track their progress.

14. What is your overall opinion of the tracking features available through the online program?

a. PROBE: What do you like the most about the tracking features?

b. PROBE: What do you like the least about the tracking features?

c. PROBE: What do you think about different tracking features? (e.g. individual tracking, basic tracking, etc.) 
15. Do you have any suggestions for how we can improve the tracking features available through our online program?

*Show screenshot of mobile version of food and physical activity tacking tools, check-in feature, and weight tracker*

These are screenshots of what the BMIQ food and activity trackers, check-in feature, and weight tracker will look like if accessed on a smartphone or tablet.

16. What is your opinion of the mobile versions of the online weight management program's food and activity trackers, check-in feature, and weight tracker

a. PROBE: What do you feel are the most important tracking functions to have on the app version?

b. PROBE: Do you think you will use these tracking features? Why/why not? What about the web version?

c. PROBE: Do you think you'd be more likely to use the web version or app version of these trackers?

Now that you've seen all of the main features in BMIQ, we would like to ask a few questions about your overall opinion of the program.

d. Overall, what do you think about BMIQ? PROBE: Are there any features of BMIQ that stand out to you as particularly useful or not useful?

17. Do you have any other thoughts or feedback about the website or app layouts?

18. Do you have any other suggestions or recommendations for how we can modify or improve the BMIQ website or app?

\section{Population Health Management Questions (15min)}

This next set of questions will discuss the population health management component of the study. Some participants who use the online weight management program will also receive monitoring and support from a population health manager, a non-clinical staff member who works closely with the primary care practices. The population health manager will be responsible for monitoring patients' progress in BMIQ and reaching out to them at certain times to provide feedback and support.

1. Based on your experience, how involved and in what capacity would you like your primary care doctor to be in your weight management program?

2. Do you think regular check-ins about your weight with your primary care doctor or other member of the primary care practice would be beneficial?

a. PROBE: If not, would you instead prefer to contact your doctor directly if you need additional assistance or have questions? 
3. What type of feedback would you like to receive from your primary care doctor or other member of the primary care practice about your weight?

a. PROBE: At what points during the program do you think feedback from your doctor would be most helpful?

4. Would you be comfortable with someone other than your primary care doctor (e.g., a population health manager or dietitian) reaching out to you about your weight?

a. PROBE: Would you want any information discussed to be passed along to your primary care doctor? If so, what information?

b. PROBE: Scenario: If you haven't logged in to BMIQ in over two weeks, the population health manager would call to check-in on you and discuss any issues. Do you think this would be helpful?

5. How involved would you like a nutritionist or dietitian to be in your weight management program?

a. PROBE: What type of feedback would you like to receive from a nutritionist or dietitian about your weight?

b. PROBE: When/how would you prefer to interact with a nutritionist/dietician?

6. Is there anything else that you'd like to add or tell us related to anything that we talked about today?

This concludes our focus group session. If you have any additional comments or questions please feel free to ask now. Alternatively, you can contact the Principal Investigator, Dr. Heather Baer, using the information provided on your information sheet.

Once again we would like to thank you for participating in such an important part of our study. 
APPENDIX 2. FOCUS GROUP GUIDE (FOCUS GROUP 3)

GUIDE FOR THE THIRD FOCUS GROUP

Once again, we'd like to thank you for participating in this focus group. Before we start, let's go around the room and introduce ourselves.

The questions during this focus group will be about the online weight management program, BMIQ, that we will be using for our study. I will ask you some questions about the tasks that you were asked to complete on your own. Don't worry if you weren't able to complete all of the tasks; if you had trouble with any of them, we would like to hear about this.

\section{General questions:}

1. Before we start with specific questions about the features/tasks, we would like to know how you accessed the BMIQ website (desktop computer, laptop, tablet, or smartphone)?

2. Why did you decide to use a (desktop computer/laptop/tablet/smartphone) to access the website?

3. Did you use more than one approach to access the website (desktop computer/laptop/tablet/smartphone)? If yes, how was it for you?

4. In general, how easy or difficult was it to log in and use the website? 
Task 1: For task 1, you were asked to log in to the BMIQ website and look at the Home page. Were you able to do this?

YES - Continue

NO - No problem! Let's try to work through it together.

5. How easy or difficult was it to log in to the Home page?

6. What did you think about the Home page?

a. PROBE: How did it look to you? Was the information and layout clear?

b. PROBE: What did you think you could do from here?

c. PROBE: Do you have any additional comments or recommendations about the home page?

Task 2: For task 2, you were asked to click on each of the tabs across the top of the Home page to navigate to the other BMIQ pages (Sessions, To Do list, Meal Plan, etc.) Were you able to do this?

YES - Continue

NO - No problem! Let's try to work through it together.

7. How easy or difficult was it to navigate to each of the pages on the website?

8. What did you think about the navigation and layout of the site?

a. PROBE: Was the information on each page what you expected to see there?

b. PROBE: Did you have trouble with any of the tabs?

c. PROBE: Do you have any additional comments or recommendations about the navigation of the website or any of the tabs?

Task 3: For task 3, you were asked to go to the Sessions page and review the information in Session 4 (Setting Smart Goals for Weight Control), including the video. Were you able to do this?

YES - Continue

NO - No problem! Let's try to work through it together.

9. How easy or difficult was it to go to the Sessions page and review the information in Session 4 ?

10. What did you think about Session 4 and the sessions in general?

a. PROBE: Do you think the sessions are useful (written information \& video)? Why or why not?

b. PROBE: Was the information in Session 4 easy or difficult to understand?

c. PROBE: Did the Session 4 video work as expected?

d. PROBE: Do you have any additional comments or recommendations about 
Session 4 or the sessions in general?

Task 4: For task 4, you were asked to complete the To Do list for Session 4. Were you able to do this?

YES - Continue

NO - No problem! Let's try to work through it together.

11. How easy or difficult was it to complete the To Do List?

12. What did you think about the To Do List?

a. PROBE: Do you think the To Do List is useful? Why or why not?

b. PROBE: Do you have any additional comments or recommendations about the To Do List?

Task 5: For Task 5, you were asked to go to the Meal Plan page and look at the information there, including the sample menus and meal exchange information. Were you able to do this?

YES - Continue

NO - No problem! Let's try to work through it together.

13. How easy or difficult was it to go to the Meal Plan page and look at the information?

14. What did you think of the Meal Plan page?

a. PROBE: Do you think the Meal Plan page is useful? Why or why not?

b. PROBE: What did you think about the sample menus? Were they easy or difficult to understand?

c. PROBE: What did you think about the information about exchanges? Was it easy or difficult to understand?

d. PROBE: Do you have any additional comments or recommendations about the Meal Plan page? 
Task 6: For task 6, you were asked to go to the Weight page and enter your weight for at least two dates. Were you able to do this?

YES - Continue

NO - No problem! Let's try to work through it together.

15. How easy or difficult was it to enter your weight?

16. What did you think of the Weight page?

a. PROBE: Do you think the Weight page is useful? Why or why not?

b. PROBE: What did you think about the information displayed in the graph and the Weight History? Was it easy or difficult to understand?

c. PROBE: Do you have any additional comments or recommendations about the Weight page?

Task 7: For task 7, you were asked to go to the Food \& Activity page and enter your food intake and physical activity for one day. Were you able to do this?

YES - Continue

NO - No problem! Let's try to work through it together.

17. How easy or difficult was it to enter your food and physical activity information?

18. What did you think of the Food \& Activity page?

a. PROBE: Do you think the Food \& Activity page is useful? Why or why not?

b. PROBE: What did you like or not like about entering your food and physical activity information?

c. PROBE: Do you have any additional comments or recommendations about the Food \& Activity page? 
Task 8: For task 8, you were asked to go to the Resources page and look at some of the materials there, including the welcome letter, articles, and videos. Were you able to do this? YES - Continue NO - No problem! Let's try to work through it together.

19. How easy or difficult was it to access the information on the Resources page?

20. What did you think of the Resources page?

a. PROBE: Do you think the Resources page is useful? Why or why not?

b. PROBE: What did you think about the Welcome Letter? Was it easy or difficult to understand?

c. PROBE: What did you think about the articles? Were they easy or difficult to understand?

d. PROBE: What did you think about the videos? Were they useful?

e. PROBE: Do you have any additional comments or recommendations about the Resources page?

\section{Overall:}

21. Now that you've had a chance to look at the BMIQ website, do you think it would be something that you'd use and stick with? Why or why not?

22. Would you use all the existing features in this online program or only some of them?

23. Taking in consideration that the program will be 12 months, how many times a day or a week do you think you would log in to the website? (review materials, track, etc.)

24. Did working through the tasks make you think about your weight/diet at all? How so? Did it motivate you at all to want to make a change?

25. Do you have any other thoughts or feedback about the website's content, layout, or usability that we have not discussed so far?

a. PROBE: Do you have any other suggestions or recommendations for how we can modify or improve the BMIQ website?

This concludes our focus group. If you have any additional comments or questions, please feel free to ask them now. Alternatively, you can contact us using the phone number or email address in the information that you received by mail. If you no longer have that information, I am happy to provide it now as well. Once again we would like to thank you for participating in this focus group. 
APPENDIX 3. HEALTHCARE PROVIDERS' INTERVIEW GUIDE

HEALTHCARE PROVIDERS' INTERVIEW GUIDE

Partnerships for Reducing Overweight and Obesity with Patient-centered Strategies (PROPS)

Dr./Mr./Ms. thank you for taking the time to speak with us and agreeing to participate in this study.

We are researchers affiliated with Brigham and Women's Hospital, and are conducting a study on attitudes and perceptions of various stakeholders about our research study, "Partnerships for Reducing Overweight and Obesity with Patient-centered Strategies (PROPS)."

As you may already know, the goal of this project is to adapt an evidence-based online weight management program and integrate it with population management support from primary care practices. We then will conduct a three-arm, cluster-randomized trial to compare the effectiveness of: 1) usual care, 2) a stand-alone online weight management program, and 3) a combined intervention (online weight management program plus population management support), among overweight and obese primary care patients with type $\mathbf{2}$ diabetes or hypertension. In order to conduct our study, we need to refine and test our intervention tools and approaches before we begin implementation. We believe that your perspectives are integral to our efforts to improve the intervention.

We would appreciate you taking the time to participate in this 15-30 minute interview. You will receive $\$ 50$ for participating. The questions will focus on management of overweight and obesity and your opinion of our intervention. Your participation is voluntary, and whether or not you participate will have no effect on your employment status. You may choose not to continue the interview at any point. We want to emphasize that your answers will be kept confidential and anonymous. We want to encourage you to speak openly about your ideas.

Are you willing to participate in this interview? $\quad \square$ Yes $\square$ No

With your permission we will be recording this session. We are doing this because we want to make sure that we remember everything that you say. Your comments are really important to us. Your responses are confidential and will be used for research purposes only.

May we record this conversation? $\quad \square$ Yes $\square$ No

If not, we will take written notes to document the conversation.

Do you have any questions before we begin? If you have further questions regarding this study at any time later, please feel free to ask me or the Principal Investigator, Dr. Heather Baer.

Thank you for your cooperation, 


\section{GENERAL QUESTIONS}

1. How would you describe your main role in the organization or practice?

2. Do you know whether your organization or practice currently has weight management programs or initiatives for patients? If yes, please elaborate.

3. Are you currently involved with these management programs or initiatives? If yes, please elaborate.

4. In general, to what extent do you find these weight management programs useful?

5. Are you aware of other weight management programs that your patients are using outside of your organization?

a. What are your attitudes towards these weight management programs?

b. Are you referring your patients to other weight management programs outside of your organization? Why or why not?

c. Are you comfortable referring your patients to these outside programs? Why or why not?

6. What has been a facilitator / made it easier for you when addressing overweight, obesity, or weight management with your patients?

7. What kinds of barriers do you typically face when addressing overweight, obesity, and weight management with your patients? (probe for: counseling/advising patients, referral process, prescriptions/surgery, etc.)

a. What could help / assist you with this?

8. What could help / assist you with helping patients with weight management issues?

a. What additional services or resources would be useful for you or your patients?

9. In general, how do you feel about online weight management programs, websites, or apps? Do you typically recommend these to your patients? If so, which ones do you recommend?

a. Would you be comfortable referring your patients to an online weight management program? Why/Why not?

\section{PROGRAM QUESTIONS}

1. In general, what do you think about the interventions proposed for this study?

a. The online program

b. The population health manager component

c. Any additional intervention components

2. What do you think your involvement would be?

3. What information as a [clinician / stakeholder] would you want to receive about your patient?

4. How would you want to receive this information (e.g., could be on a printed paper, or via email, or in Epic, in person, etc.)?

a. If it were possible, would you want to have direct access to the online weight management program, so that you could see how your patients are doing?

b. Would you feel comfortable if your patients could communicate with you via the online weight management the program? Why or why not?

5. How frequently would you like updates about your patients?

6. What type of outreach do you think would be helpful for your patients?

7. What involvement should other providers have [e.g., dietician, population health manager, nurse]?

8. Any additional comments? 